\title{
TIN Meets CAD - Extending the TIN Concept in GIS
}

\author{
Rebecca, O.C. Tse and Christopher Gold \\ Department of Land Surveying and Geo-Informatics \\ Hong Kong Polytechnic University, Hung Hom, Kowloon, Hong Kong SAR \\ Tel: (852) 2766-5955; Fax: (852) 2330-2994 \\ Rebecca.Tse@polyu.edu.hk, christophergold@voronoi.com
}

\begin{abstract}
Extending the current "2.5D" terrain model is a necessary development in GIS. Existing 2D surface information must be extended to 3D, while trying to preserve the topological integrity. Computer-Aided Design (CAD) on the other hand is often fully $3 \mathrm{D}$, which may be excessive for our "extended 2D" mapping needs. We have used the "boundary representation" (brep) design from CAD systems, based on manifold models, to address these problems. Triangulated Irregular Network (TIN) models of the terrain surface are well known in Geographic Information System (GIS), but are unable to represent cliffs, caves or holes, which are required to represent complex buildings. The simple TIN b-rep structures may be extended using formal Euler Operators to add simple CAD functionality while guaranteeing the connectivity required for terrain modelling. We believe this provides a simple and reliable extension that is sufficient for many applications.
\end{abstract}

\section{Introduction}

Within the world of GIS the TIN has been well known for more than 20 years. It is one of the basic models for representing digital terrain. A piece of land on the earth's surface can be modelled as a terrain [3, 10]. Applications of TINs to problems of runoff, etc., have also been well studied in the computational geometry literature [13]. The difficulties with this "2.5D" model are also well known, in particular the fact that the structure itself is only two-dimensional, the third dimension being only an attribute. This limitation contrasts strongly with the situation in the CAD world, as well as in 3D graphics, where surface meshes of complex 3D objects (plausible and implausible) are routinely generated. What is the big problem?

It is fairly clear that GIS is still firmly two-dimensional in its implementation, despite various attempts to extend it, and despite frequent complaints about the inability to model non-planar networks, bridges, caves, etc. within the traditional TIN surface model. This paper attempts to alleviate this problem somewhat, by moving gently towards an extended view of the traditional TIN. The first step is to recognise that a terrain surface modelled by a TIN is not a two-dimensional entity - it is an air(or water-) earth interface, the boundary between the "Polyhedral Earth" and the 
exterior. Simply put: every TIN has an "underneath". This causes few difficulties in practice.

Once this polyhedral model is accepted, attention naturally turns to other disciplines' experience in polyhedral modelling. Computational geometry has developed a variety of tools for managing surface models [12]. In particular, we have been interested in the Quad-Edge structure [4, 5]. Computer graphics workers have developed many tools used extensively today. In particular, the field of CAD has been developing tools for solid model representation for many years, for example Baumgart [1] and Mantyla [7, 8].

In our current work we are using the CAD-type b-rep structure and Euler Operators to create a connected TIN model with holes (bridges or tunnels). Starting with the well-known TIN model, we have built a set of CAD-type Euler Operators, including the ability to form holes, which are easy to implement with the Quad-Edge structure [5]. Secondly we show that all basic TIN modification operations may be performed with Euler Operators. Thirdly, we show that additional Euler Operators may be used to modify the surface form in various ways, including the insertion and deletion of holes that give the basic forms of bridges and tunnels.

Thus our work consists of four stages:

- Definitions of three levels of operators to achieve our desired system;

- The use of Quad-Edge structures to implement CAD-type Euler Operators;

- The use of Euler Operators to implement basic triangulation functions;

- The extension of our triangulation models with additional Euler Operators.

\section{TINs and CAD}

The examination of CAD modelling techniques does not seem attractive at first sight. The intersection of cubes and tubes, as in Constructive Solid Geometry (CSG) modelling, hardly applies to terrain models. Modern non-manifold models of surfaces can have astoundingly complex data structures [6], and would be massive overkill for TIN enhancement. However, the simpler manifold-based "b-rep" CAD models appear to be feasible for our purposes. In particular, the careful specification of "EulerOperators" that guarantee to preserve the topological validity of the bounding surface seems particularly appropriate. Euler Operators ensure the integrity of boundary models. As stated by Mantyla [8], "in a "b-rep" model, an object is represented indirectly by a description of its boundary. The boundary is divided into a finite set of faces, which in turn are represented by their bounding edges and vertices." According to his definition, b-reps are best suited for objects bounded by a compact (i.e. bounded and closed) manifold. However, if the main concern were building a non-manifold object, it would be better to use other models.

In 1988 Weiler [14] proposed non-manifold operators to manipulate topological data in non-manifold models. Moreover, they were not based on the basic EulerPoincare formula, although some of the topological relationships still can be generalised by a new formula by Masuda [9], although it is more complicated. Nevertheless, it may be valuable to extend the b-rep with Euler Operators into a non- 
manifold GIS model in the future. We conclude that to extend the TIN, the b-rep model is the most appropriate at present.

\section{Simplicity of Implementation - the Quad-Edge Structure}

We have examined the basis for various sets of Euler operators mentioned in the CAD literature, and their data structures. This is usually the "Winged-Edge" data structure [1], which has a variety of supporting pointer structures, some of which, upon closer examination, are not necessary in order to maintain a TIN or triangulation-based b-rep surface model. In particular, if all faces are triangles, then no holes may be created in the interior of individual faces and thus some elements of both the Winged-Edge structure and the classical Euler Operators may be removed. We have used the QuadEdge structure of Guibas and Stolfi [5]. More particularly, the Quad-Edge structure has been used to implement a set of Euler Operators that have been shown to suffice for the maintenance of surface triangulations. According to Weiler [13], if a topological representation contains enough information to recreate nine adjacency relationships without error or ambiguity, it can be considered a sufficient adjacency topology representation. These Euler Operators form the basis of the standard (twodimensional) incremental triangulation algorithm. In addition, Euler Operators can serve to generate holes within our surfaces, thus permitting the modelling of bridges, overpasses etc. that are so conspicuously lacking in the traditional GIS TIN model. The individual Quad-Edge and Euler Operators take only a few lines of code each. "Make-Edge" and "Splice" (Figs. 1 \& 2) are the two simple operations on the QuadEdge structure, which is formed from four connected "Quad" objects, using the simple implementation of [4]. Every Quad has three pointers:

- N - link to next Quad ("Next") anticlockwise around a face or vertex

- R - link to next $1 / 4$ Edge ("Rot") anticlockwise around the four Quads

- $\mathrm{V}$ - link to vertex (or face)

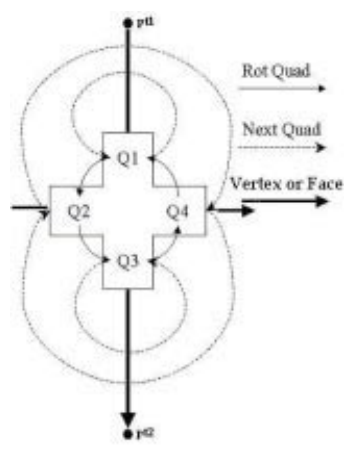

Fig. 1. Make-Edge

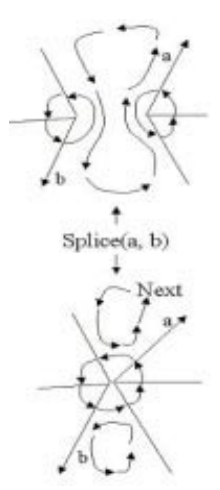

Fig. 2. Splice

The Make Edge operator creates a new independent edge. The Splice operation, which is its own inverse, either splits a face loop and merges two vertex loops (upper 
to lower parts of Fig 2) or else splits a vertex loop and merges two face loops (lower to upper). This operation suffices to maintain any connected graph on an orientable manifold, such as is the case for CAD b-rep models, and TINs.

\section{Selection of a Set of Euler Operators}

According to Braid [2], five spanning Euler Operators (plus the Euler-Poincare formula) suffice to specify the number of elements in any b-rep model. Conversely, the inverse formulation serves to identify the number of Euler Operators needed to build a specified model, and to validate its topological integrity. The six elements of the solid model are: vertices; edges; faces; loops (or rings); holes; and bodies (or shells), and are usually based on the Winged-Edge structure.

However, in TINs there are no loops (holes in individual faces), so these will not be considered, leaving five elements. Thus four spanning Euler Operators suffice for TINs with holes. In the traditional TIN models there are no holes, so three Euler Operators will serve for our initial model, to be extended to four in section 7.

\section{Implementation of Euler Operators using Quad-Edge structures}

The three initial Euler Operators are described below. "Make Edge Vertex Vertex Face Shell" (MEVVFS) creates the initial shell, and "Kill Edge Vertex Vertex Face Shell" (KEVVFS) removes it. "Make Edge Face" (MEF) and its inverse "Kill Edge Face" (KEF) create or kill an edge and a face. "Split Edge Make Vertex" (SEMV) splits an edge, and its inverse is "Join Edge Kill Vertex" (JEKV). While other operators are possible, this set of operators is appropriate for a triangulation model, where no "dangling edges" are permitted.

\subsection{MEVVFS $\Leftrightarrow$ KEVVFS}

"MEVVFS" adds an edge, two vertices, one face and one shell to an empty model. Its inverse "KEVVFS" removes them. One Quad-Edge Operator is used to implement "MEVVFS" (which is simply "Make-Edge") to create a single edge. In our approach the "Loop" [7, 8, 9] around the single edge is really the back face of our model, so we consider this to be a face.

\subsection{MEF $\Leftrightarrow$ KEF}

"MEF" and "KEF" are used to create an edge and a face, and to delete them. In "MEF" we need to give two quads as parameters to make a new face. In "KEF" we need to give an edge as a parameter for removing the edge, and one face will be destroyed as this edge is removed. Fig. 3 shows the operation of "MEF" and "KEF". 
"MEF" inputs two quads "a" and "b", output is one new quad "e". "KEF" removes an edge "e" using two splices and one face is killed.

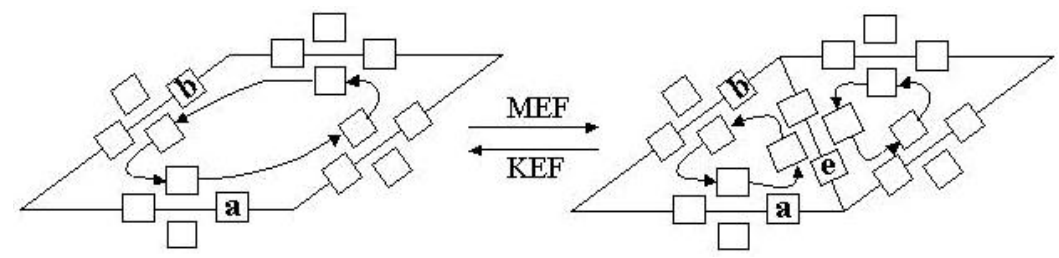

Fig. 3. "MEF" $\Leftrightarrow$ "KEF"

\subsection{SEMV $\Leftrightarrow$ JEKV}

We use "SEMV" and "JEKV" to split one edge into two pieces. This procedure adds or removes a point on an edge. It is useful for creating triangles without creating a dangling arc. Fig. 4 shows the operators of "SEMV" and "JEKV". "SEMV" splits edge "e" into two parts, and two parts are joined by "JEKV", using splice operations.

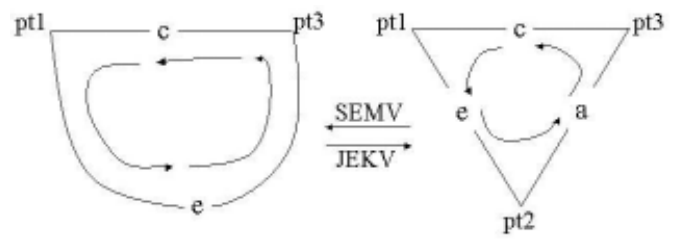

Fig. 4. "SEMV" $\Leftrightarrow$ "JEMV"

\section{Implementation of the TIN Model Using Euler Operators}

In the TIN model we have three main functions, which are: create a first triangle; insert a point; and swap an edge. We use Euler Operators to implement triangulation functions. We use the functions "Big Triangle", "Insert Point" and "Swap". These also have inverses.

\subsection{Big Triangle}

In our model we limit ourselves to complete faces with no "dangling edges", so we begin from nothing, and create the first triangle using "Big Triangle". We kill the big triangle and go back to nothing by using the inverse functions as shown in Fig. 5. In creating the "Big Triangle", three points are needed as input. Three different Euler Operators are used: "MEVVFS", "MEF" and "SEMV". 3 points (pt1, pt2, and pt3) are input to create the Big Triangle and 3 edges (e1, e2, and e3) are the output. 
"MEVVFS" creates the first edge "e1". "MEF" creates a new edge "e3". "SEMV" splits edge "e3". To kill the Big Triangle, "JEKV" joins edge "e2" and "e3". "KEF" kills edge "e3" and a face. "KEVVFS" kills the last edge "e1", giving an empty space.

\subsection{Insert Point}

"Insert point" is another procedure that we use. In the TIN model the whole surface is formed by triangles, therefore we will insert a new point into an existing triangle. We can use "MEF", "SEMV" and "MEF" to insert the new point, and "KEF", "JEKV" and "KEF" to delete the point. Fig. 6 shows the procedure of inserting a new point. Inserting a new point makes three edges and two faces. "MEF" creates an edge "N4". "SEMV" splits an edge "N4". Last step "MEF" creates a new edge "N6".

\subsection{Swap}

Swap is a procedure for swapping two edges inside the TIN model. For the Delaunay Triangulation, we use the "in-circle" test to test the triangle, and use the swap operator to change edges. The Delaunay Triangulation is based on the empty circumcircle criterion [5]. It has been accepted that the Delaunay Triangulation is the best criterion to use for triangle definition. Fig. 7 shows the steps for Swap using Euler Operators. We need to input an edge "e" to be changed. "KEF" kills the edge and "MEF" creates the edge. It swaps the edge between two triangles.

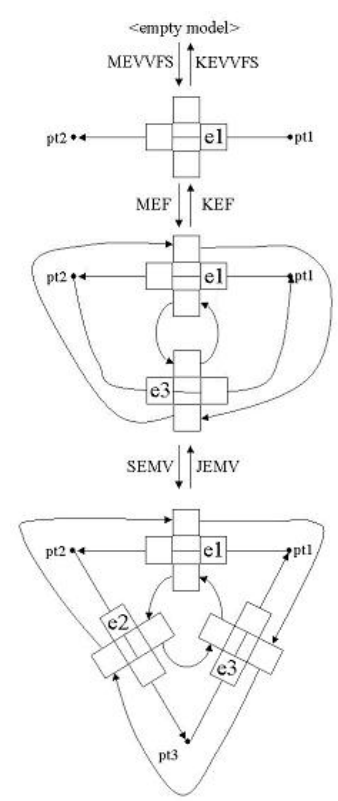

Fig. 5. "Big Triangle"

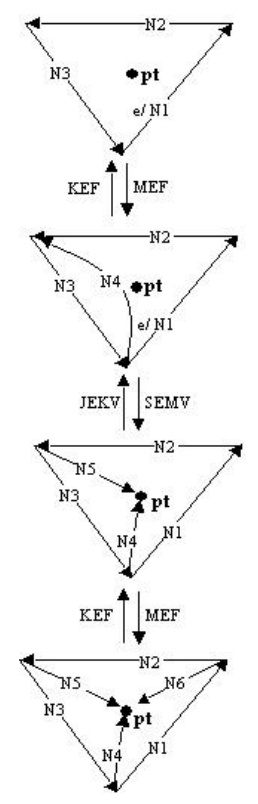

Fig. 6. "Insert Point"

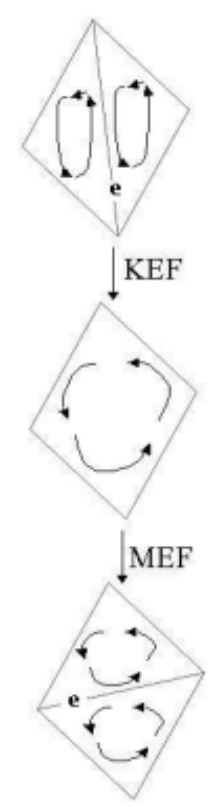

Fig. 7. "Swap" 


\section{Extending the TIN Model Using Euler Operators}

So far we have merely re-formulated the traditional incremental algorithm, but with this background we can use existing Euler Operators to modify the TIN surface in other ways, e.g. by the insertion and deletion of holes. The operator "Make Edge Hole Kill Face" (MEHKF) consists of exactly the same code as "MEF" [11]. However, instead of taking two Quad-Edges that are part of the same face loops as parameters, it takes Quad-Edges that are parts of separate face loops. The two triangles concerned are deleted, an edge is formed between the two triangles, and a new face is formed that loops through each of the deleted triangles and both sides of the new edge.

We select two triangles in an existing TIN model: their edges are ordered anticlockwise from outside. Two original triangles from the TIN model are shown, looking from "inside" in Figs 10-12. The order of the three edges inside the two triangles is $1=>2=>3 \Rightarrow>1$ and $4 \Rightarrow 5 \Rightarrow 6=>4$. We make a new edge between these two triangles and a hole is created. One face loop is created inside the hole and two triangle faces are killed.

Fig. 10 shows the result of the first step "MEHKF". The connection of the edges will be $1=>P=>5 \Rightarrow 6=>4=>Q=>2 \Rightarrow 3=>1$. Fig. 11 shows the result after performing "MEF". One new face is made and the connectivity of the edges will be 1 $=>P=>5 \Rightarrow R=>1$ (New face) and $3 \Rightarrow S=>6=>4 \Rightarrow Q=>2=>3$. Fig. 12 shows the result of the final "MEF". One new edge and face are created. There are now three faces inside the hole, but the connectivity is preserved and you can walk through the hole. We have therefore shown that the elementary Quad-Edge based Euler Operators are able to generate and modify the traditional TIN structure, permitting basic CAD operations. Three more "MEF"s are used to split the faces inside the hole into triangles. Fig. 13 shows the top view of a TIN model. Fig. 14 shows a hole and a bridge on the TIN.

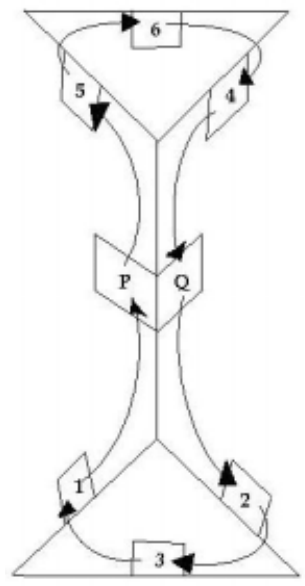

Fig. 10. "MEHKF"

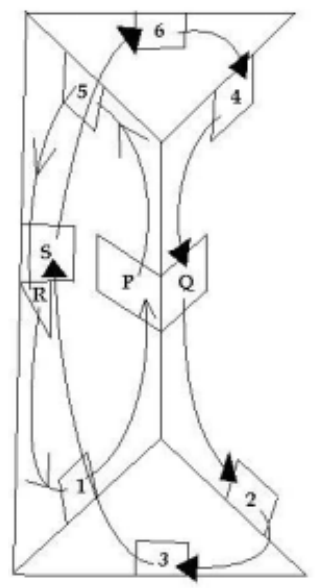

Fig. 11. "MEF"

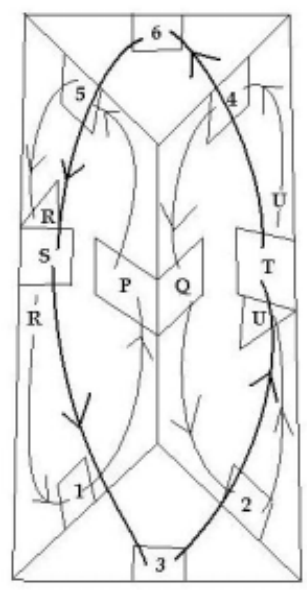

Fig. 12. Third "MEF" 


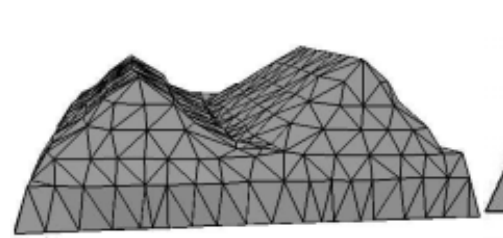

Fig. 13. Top view of TIN model

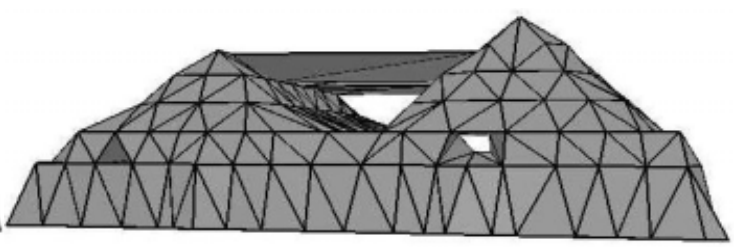

Fig. 14. A Hole and a bridge on the TIN

\section{Relation to Previous Work}

The work we describe here has several steps, each of them new within a particular context:

1) Euler Operators, as used in CAD systems, are usually based on the Winged-Edge structure of [1]. The implementations of [7,8] indicate the complexity of this approach. We show here that the Quad-Edge structure of [5] is a more natural, and much simpler, methodology. This is of potential interest to the CAD and Computer Graphics communities.

2) For the GIS community, we show the close relation between TIN modelling, brep structures from $\mathrm{CAD}$, and Computer Graphics mesh generation. By eliminating one $\mathrm{CAD}$ primitive (holes in faces) and restricting ourselves to triangular elements, Euler Operators may be directly ported to GIS.

3) Once TIN operations are expressed as Euler Operators, additional topological operations may be performed directly, in particular the creation and deletion of holes permitting the interactive formation of bridges, tunnels, etc. To our knowledge, the introduction of some CAD techniques into TIN modelling has never been suggested before.

\section{Conclusions and Acknowledgements}

Thus, in summary, we are following a CAD-based b-rep sense of connectedness that uses Euler Operators to build the well-known TIN model, and extends it to permit holes and caves - all based on the Quad-Edge structure. We show the validity and the implementation of these operators in preserving the connectedness of our " $2.75 \mathrm{D}$ " TIN model. Operational details concerning the navigation within the mesh, and the selection of individual elements, have not yet been finalized. Nevertheless, the results conform well to the basic concepts of model representation within OpenGL, they depend on very similar data structures to those currently available for TINs, and they greatly simplify the implementation of a basic CAD system. We feel that this juxtaposition of computer science, CAD and GIS techniques opens up a variety of interesting possibilities, not least the manipulation of terrain models to represent some of the more complicated forms of human landscape modification. 
Having now based our TIN model on a small set of simply-implemented Euler Operators, we are free to follow the CAD approach and build additional high-level functions, to form specific features (e.g. buildings) in our triangulation structure.

The authors would like to acknowledge the partial support of this research by Research Grants Council, Hong Kong SAR, project B-Q405.

\section{References:}

1. Baumgart, B.G. (1972): Winged edge polyhedron representation. Stanford University Computer Science Department, Stanford Artificial Intelligence Report No. CS-320.

2. Braid, I.C., R.C. Hillyard, and I.A. Stroud: Stepwise Construction of Polyhedra in Geometric Modelling. In: Mathematical Methods in Computer Graphics and Design, Ed K.W. Brodlie: Academic Press of Computer Laboratory, University of Leicester, Leicester, England, pp. 123-141.

3. Gold, C., (1979): Triangulation-Based Terrain Modelling-Where are we now? Proceedings: Auto-Carto 4, International Symposium on Cartography and Computing; Baltimore, MD, 1979, pp. 104-111.

4. Gold, C., (1998): The Quad-Arc Data Structure. Proceedings: 8th International Symposium on Spatial Data Handling; Vancouver, BC, pp. 713-724.

5. Guibas, L., and J. Stolfi (1985): Primitives for the Manipulation of General Subdivisions and the Computation of Voronoi Diagrams. ACM Transactions on Graphics, Vol. 4, No. 2, pp. 74-123.

6. Lee, K. (1999): Principles of CAD/ CAM/ CAE Systems. Seoul National University. Korea.

7. Mantyla, M. (1981): Methodological Background of the Geometric Workbench. Helsinki University of Technology, Finland.

8. Mantyla, M (1988): An Introduction to Solid Modeling. Helsinki University of Technology, Finland.

9. Masuda, H., Shimada, K., Numao, M., and Kawabe, S. (1990): A Mathematical Theory and Applications of Non-manifold Geometric Modelling. In: Advanced Geometric Modeling for Engineering Applications North-Holland, Amsterdam

10. Peucker, T. K., R. J., Fowler, J. J., Little, and D. M., Mark, 1978: The triangulated irregular network. In: Proceedings Digital Terrain Models Symposium (St. Louis: American Society for Photogrammetry), pp. 516-532.

11. Tse, O.C., and C. Gold (2001): Terrain, Dinosaurs and Cadastres - Options for ThreeDimensional Modelling. Proceedings, International Workshop on "3D Cadastres", Delft, Netherlands, November, 2001, pp. 243-257.

12. van Kreveld. M. (1997): Digital elevation models and TIN algorithms. In: M. van Kreveld, J. Nievergelt, T. Roos, and P. Widmayer (eds.). Algorithmic Foundations of Geographic Information Systems, number 1340 in Lecture Notes in Computer Science (tutorials), pp. 37--78. Springer-Verlag, Berlin.

13. Weiler, K. J. (1986): Topological structures for geometric modeling. PhD thesis, Rensselaer Polytechnic Institute, University Microfilms International, New York, U.S.A.

14. Weiler, K. J. (1988): Boundary Graph Operators for Nonmanifold Geometric Modeling Topology Representations. In: Geometric Modeling for CAD Applications, Elsevier Science, Amsterdam.

15. Zeid, I., (1991): CAD/ CAM theory and practice. McGraw-Hill, New York, pp.368-388 